\title{
An effective Teaching/Learning Intervention on Time Dilation and Relativistic Dynamics
}

\author{
Emanuele Pugliese*, Lorenzo Santi* \\ *Department of Chemistry, Physics and Environmental Sciences, \\ Udine University, 206 Via delle Scienze, Udine, UD 33100 - Italy
}

\begin{abstract}
An educational path on relativistic dynamics has been designed and experimented in an upper-secondary school class to introduce essential concepts for making meaning of mass-energy equivalence. The pathway aims at building conceptual understanding of relativistic linear momentum, kinetic energy and their interrelation. It draws on relativistic invariant quantities and exploits thought experiments as well as educationally relevant ones; it illustrates both scientific modeling by induction both hypothetical-deductive methods for assuring logical consistency. Rough new technologies were used: an on-line simulation of light-clock and timed animations of two-particle relativistic collision. Learning and understanding were assessed by both comparing the administered pre- and post-test both analyzing the latter. General interpretive questions were asked during the teaching/learning process in order to find out pupils' intermediate explanatory models and problem solving skills in similar contexts. An analysis of the interplay among prior, posterior and intermediate answers is presented here. The results indicate conceptual change for kinetic energy and vacuum light-speed. Only understanding of time interval dilation effect was detected instead.
\end{abstract}

Keywords: Relativistic momentum, relativistic kinetic energy, time dilation, light-clock on-line simulation, timed animations, representation construction, conceptual understanding, conceptual change.

PACS: 01.40.Fk; 01.40.ek.

\section{INTRODUCTION}

Teaching and learning Special Relativity has not been extensively explored in physics education research yet, as recently pointed out [1]. Some works were carried out on basics and/or kinematics [2 - 6], but dynamics seems to have been much neglected, in particular those subjects directly involved in deducing and making meaning of mass-energy equivalence. Exceptions are Relativistic Concept Inventory [7] and attempts to deduce momentum [8] and energy [9].

Taking into consideration relativistic dynamics is the intention of the present work, in order to let highschool students understand its conceptual nuclei through a logically consistent teaching/learning path. The ultimate aim is let pupils construct personal meaning-making of this seemingly "exotic" matter.

The choice of this topic is coherent with the new syllabus indications for Italian secondary school's last year, in which modern physics is mandatory, with an emphasis on mass-energy dynamic relationship and its scientific and technological spinoffs.

A wide educational literature has demonstrated the importance of probing single student's learning in progress [10], so pupils underwent pre- and post-test and two groups of intermediate questions.

The first research aim was seeking for intermediate concepts/models not only in propositional form, but also as images, episodes, intellectual and motor skills that a student associated with a certain label [11]. The second objective was studying the sample's conceptual ecology evolution [12]. The target physics content consisted of time interval invariance, expressions for dynamic quantities at high speed and role of vacuum light speed in mechanics.

\section{RATIONALE}

Experimental evidence that a limiting speed does exist in nature and, if limit, (a) it has to be invariant was examined by summarizing Bertozzi's results [13] on electron acceleration up to $15 \mathrm{MeV}$ kinetic energies in 1964; then (b) the Relativity Principle was assumed both in theoretical and experimental form for all physics. The two postulates were thus stated. After clock synchronization problem in a single frame, the relationship between time intervals in different frames was afforded: students visualized a simulation for constructing a mental qualitative representation of time dilation effect. Its formula was then deduced by the Pythagorean Theorem in the well-known light-clock consensus platonic thought experiment [14]. Because of work-energy theorem $\Delta p\langle u\rangle=\Delta K$, relativistic momentum needs to be changed too.

It was derived in a teaching meditative constructive thought experiment [14], inspired by Fabri [15], Taylor $\&$ Wheeler [16] and Feynman [17].

A relativistic collision between two identical particles was studied in the center-of-mass (CM) and 
'laboratory' (K) inertial reference frames, the second being defined so that one particle is moving transversally to the direction of the two frames' relative translation at non-relativistic speed. This was obtained by means of time dilation and its paradoxical side-effects on the relativistic particle speed in $\mathrm{K}$.

The thought experiment draws on invariance of transverse displacements, transverse total momentum and proper time; Correspondence Principle is invoked to connect relativistic momentum to classical one.

The factor $\gamma$ came out to be a relevant function in relativistic mechanics, so it was used to induce kinetic energy expression by selecting and plotting Bertozzi's data, together with a new one coming from a physical constraint. Then an appropriate interpolating linear function was found out and its Newtonian limit was taken both (i) for generalizing the obtained local result both (ii) for determining the theoretical value of the approximate best-fit line slope.

\section{INSTRUMENTS AND METHODS}

The students filled in a pre- and almost identical ${ }^{1}$ post-test (table 1). The latter was used for detecting their acquired knowledge and ways of arguing. Pre/post comparison was a useful assessment of the conceptual change - which means learning science in constructivist perspectives - between initial and final state in terms of framework theory restructuring [18] toward scientific concepts and, secondarily, assessment of ways of arguing too. Moreover, after each selfstanding instructional unit intermediate interpretive written tasks were administered to probe how, if so, the expected reorganization occurred $[12,18]$.

Sound mental representations are known to entail «connections between ideas, artefacts, representations and contexts» as well as development of pliable problem-solving skills [19], thus a concept map and conceptual problems in similar, but different, situations were administered: questions $4(Q 4)$, and $5(Q 5)$ in table 2. Cognitive transfer is detected by these tools.

A visual approach has been tried out, exploiting simple new technologies, owing to the present emphasis on image study in much contemporary science and school science accordingly [19].

In this connection Gilbert [20] reaffirmed the centrality of visualization to science learning owing to the importance of understanding how to move within and between different modalities of representation.

The class visualized an on-line applet representing two mirror boxes with a light ray inside travelling back and forth, in order both to establish an operative definition of time through a periodic phenomenon and to compare light paths in presence of relative box speed. Rough timed animations were then used for the collision, for stressing (i) the symmetry and simplicity of its description in $\mathrm{CM}$ and (ii) the paradoxical 'slowness' of the relativistic particle in $\mathrm{K}$ frame.

TABLE 1. Pre- and post-test questions Question Text

\begin{tabular}{|c|c|}
\hline & Question Text \\
\hline 1 & $\begin{array}{l}\text { "Consider an isolated physical system (body) moving } \\
\text { unsteady" [omissis] In general, is there a physical } \\
\text { quantity which is conserved, that is, which remains } \\
\text { constant over time? Which one? Justify your answer" }\end{array}$ \\
\hline 2 & $\begin{array}{l}\text { "Kinetic energy is: 1) The quantity expressed by the } \\
\text { equation } \mathrm{K}=1 / 2 \mathrm{~m} \mathrm{v}^{2} \text {; 2) The form of energy associated } \\
\text { with a particle/mass-point's motion state; 3) A } \\
\text { contribution to total energy of a moving physical } \\
\text { system to be considered for testing energy conservation; } \\
\text { 4) A combination of the above (specify which one). } \\
\text { Justify your choice" }\end{array}$ \\
\hline 3 & $\begin{array}{l}\text { "Does work-energy theorem imply that if a work is } \\
\text { done on a body its velocity and/or kinetic energy can be } \\
\text { limitless increased? Explain" }\end{array}$ \\
\hline 4 & "Does the same hold for linear momentum? Explain" \\
\hline $5 \mathrm{a}$ & $\begin{array}{l}\text { "Suppose that you observe on a train an object moving } \\
\text { at } 10 \mathrm{~m} / \mathrm{s} \text { with respect to you. Your mate on the station } \\
\text { platform is estimating that the train moves at } 25 \mathrm{~m} / \mathrm{s} \\
\text { relative to him in the same direction of object's motion. } \\
\text { What is the object speed in the inertial frame of the } \\
\text { platform? What formula did you apply to get it?" }\end{array}$ \\
\hline $5 b$ & $\begin{array}{l}\text { "Suppose that you observe on a train a propagating } \\
\text { laser beam [the rest of the text is completely analogous } \\
\text { to that of } 5 a \text { ]. Explain how you get your result." }\end{array}$ \\
\hline 6 & $\begin{array}{l}\text { "[omissis] Is duration of a phenomenon, such as the fall } \\
\text { of something to the ground, the same for all observers? } \\
\text { Reply and explains the assumptions made" }\end{array}$ \\
\hline 7 & $\begin{array}{l}\text { "[omissis] Which of the following quantities are } \\
\text { invariant and why? Time interval (duration); Kinetic } \\
\text { energy; Momentum; Length; Light speed in vacuo; } \\
\text { Speed of a sound produced by one observer; Mass" }\end{array}$ \\
\hline
\end{tabular}

TABLE 2. Intermediate questions

\begin{tabular}{c|l}
\hline \multicolumn{1}{c}{ TABLE 2. Intermediate questions } \\
\hline & \multicolumn{1}{c}{ Question Text } \\
\hline 1 & $\begin{array}{l}\text { "Why do we conclude that work-energy theorem is } \\
\text { valid at high speeds too? Explain" }\end{array}$ \\
\hline 2 & "List main outcomes from Bertozzi's experiment" \\
\hline 3 & $\begin{array}{l}\text { "Why do we synchronize clocks with a light signal } \\
\text { and not of another type, such as sound?" }\end{array}$ \\
\hline 4 & $\begin{array}{l}\text { "An observer on a train and another on the station } \\
\text { platform were given two identical clocks. Are first } \\
\text { observer's heartbeats really slowed down for the } \\
\text { other? Does the second observer feel his own } \\
\text { heartbeats slowed down? Explain" }\end{array}$ \\
\hline 5 & $\begin{array}{l}\text { "A bridge manipulator and a passenger on a train } \\
\text { moving on a movable bridge were given two identical } \\
\text { clocks. Is time interval between bridge opening and } \\
\text { closing different for the train passenger with respect } \\
\text { to the bridge manipulator? Explain" }\end{array}$ \\
\hline
\end{tabular}

\footnotetext{
${ }^{1}$ The only difference is between $5 a$ (pre-test) and $5 b$ (post-test).
} 


\section{DATA ANALYSIS AND RESULTS}

Qualitative content analysis was carried out. Our 'criterions of selection' [21], derived from the research questions, let us induce mutually exclusive concept classes (categories); then their frequencies were calculated. Significance level was set at 0.01.

\section{Pre/post-test comparison}

Some knowledge acquisition after the proposed instruction was highlighted by data analysis.

i. Kinetic energy (K.E.) acquired broader meanings: 'motion energy' primarily, and/or 'essential term for energy conservation'; furthermore the choices of its classical expression broke down (Fig. 1).

ii. Motivated references to K.E. e.g. "K.E. is an energy expressed by the equation $K=1 / 2 m v^{2}$ associated to particle motion" or "K.E. depends upon the body's mass and speed which it's moving at" dropped too: this frequency passed from 15/20 to 4/20.

iii. A new category stemmed: $11 / 20$ students reported that K.E. may be increased to infinity and at the same time speed be upper limited. Typical answers: "No, energy can rise without limits but speed doesn't exceed light's"; "No, as speed never exceeds c though one keeps supplying energy to the particle and rise indefinitely its K.E.

iv. An analogous narrow category (4/20) originated for momentum. Example: "Yes, since the value of $p$ can approach to $\infty$ at relativistic speeds, but the speed $v$ can't reach values over c". It's included in the previous category.

v. As for question 7, post-test, 19/20 pupils recognized $c$ as relativistic invariant and $6 / 20$ acknowledged the validity of the syllogism 'if $c$ is the speed limit, then $c$ is invariant'. Example: "Light speed in vacuo yes, it's the limit speed and therefore it has precisely the same value in every inertial frame". One-half sample answered $5 \mathrm{~b}$ using the same syllogism, for instance " $3 * 10^{5} \mathrm{~km} / \mathrm{s} \rightarrow$ Because a massive body can't exceed that speed!" or "The speed is close to c because c can't be exceeded". Using Pearson's coefficient a significant statistical correlation was found between the answers to 7 and $5 \mathrm{~b}$ in which the syllogism was $\left(r=0.65, \rho_{0}=0.57\right.$; strong effect size: $r^{2}=0.43$ ).

vi. Before the path $2 / 20$ and $3 / 20$ pupils acknowledged the existence of a speed limit in items 3,4 respectively; $15 / 20$ and 13/20 did the same after it.

vii. The answers to question 6 asserting time interval invariance went from $10 / 20$ to $1 / 20$, while the ones asserting non-invariance from $6 / 20$ to $18 / 20$.

viii. More specifically, prior to the path some conditions for measuring time intervals were added in eight answers of the first category, for instance "the response time of each person is to be calculated" or "It will have the same value if the same measure units and the same reference frames are considered". Furthermore 8/20 students focused on the process of measure, $6 / 20$ on the observers and $3 / 20$ on certain phenomenon parameters: speed of fall, attraction forces, friction and height (categories in Fig. 2). After the path, time dilation effect and time dependency upon speed are referred to in 12/20 cases overall. Typical answers for the former: "It depends on the observers' speed: if it's close to light's, time dilation will have to be considered" or "No, because time changes in different systems owing to duration dilation effect"; for the latter: "No, because the time measured by observers depends upon the travel speed and thus upon motion".

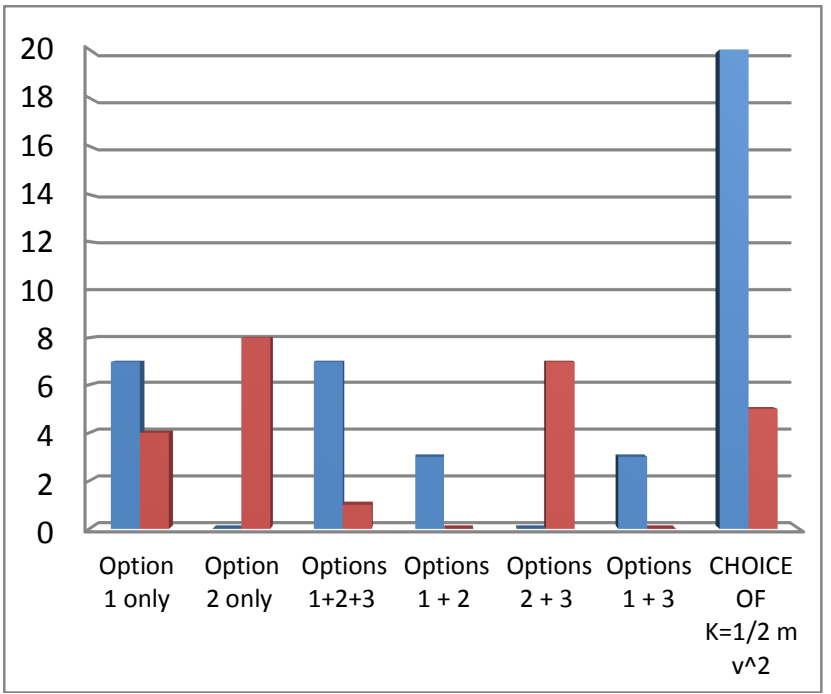

FIGURE 1. Answer category discrete distributions of pretest (left) and post-test (right) for question 2.

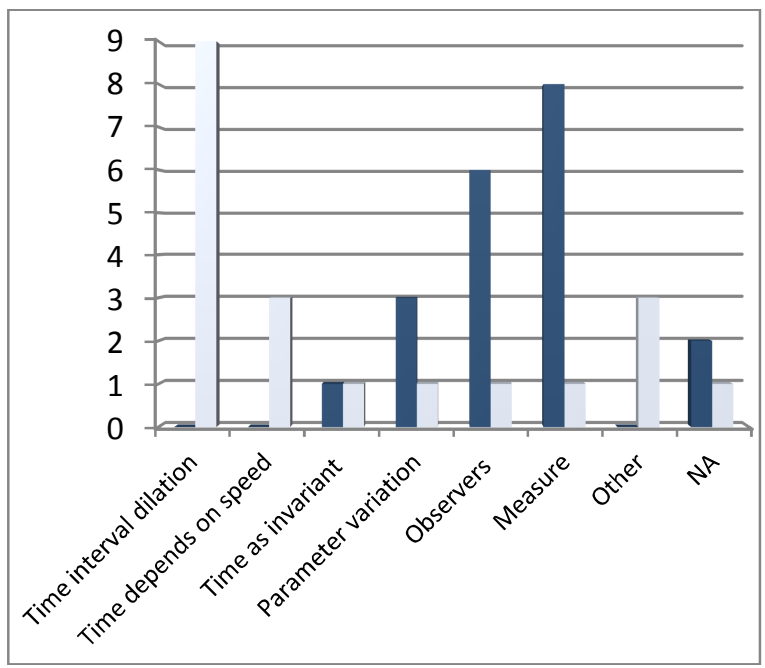

FIGURE 2. Motivation category discrete distributions in pre-test (blue) and post-test (light blue) for question 6 . 


\section{Intermediate question analysis}

23 students were present at test administration; the ones missing in the results answered off the point or didn't. $Q 4$ and $Q 5$ were meant for probing cognitive transfer skills - entangled with sound mental model formation [19] - on time dilation. Cognitive transfer occurs when a student succeeds in solving problems in which the duration of any phenomenon is observed from different frames. $Q 4$ aimed also at discriminating between descriptions of a 'distortion of perception' and a physical effect; see [1] and references for details.

Results for $Q 4$ : transfer occurred in $15 / 23$ cases, in 10 of which the effect is described as real; in $5 / 23$ no transfer was found; in $2 / 23$ we couldn't determine if it occurred. Example for real effect: "yes, since the observer is moving and thus time is slowed down. No for he's not in motion but still on the platform"; for perceptive effect: "they seem slowed down for the second but they aren't; no because he's still with respect to himself'. Q5: transfer in 11/23 answers, no transfer in $1 / 23$, uncertainty in $9 / 23$.

\section{CONCLUSIONS}

The worked-out teaching/learning path is effective for the examined class. Generalization is reasonable because a randomized sample was taken, which can be assumed as including «cases with all relevant attributes as in the population» [22]. The effect size is strong enough for the dimensions of our sample: Fischer [23] recommends $r>0.6$ for $N=20$.

Conceptual change for kinetic energy and vacuum light speed came out. Understanding of time interval dilation was detected instead in most answers ex-post.

K.E. re-assumed its primary meaning and/or the role of part of a wider conserved quantity (total energy) and at the same time it become conceptually separate from the classical expression and related in a new (scientifically correct) way to speed. Thus students can be said to properly master the concept embedded in a re-structured domain-specific knowledge framework, which is what is intended for learning in current "coherence" approach to conceptual change [18].

The same can be argued for the concepts of invariance of $c$ and existence of a speed limit. They were overall reported by at least one-half answers $e x$ post to four different items; the syllogism ' $c$ is the limit speed $\Rightarrow c$ is invariant' come out to be strongly held by some students, for it's in the answers to $5 \mathrm{~b}$ and 7 in a statistically correlate way as well as in the concept map explanation, with frequency $9 / 23$.

It may be asserted that the duration dilatation effect has been understood, but not learned. In fact most students held its mental representation after the path, but few ways of reasoning and no inclusion in a 'theory' were revealed. It might be due to the absence of any prior conceptual referent, unlike K.E. and $c$.

\section{REFERENCES}

[1] O. Levrini, "The Role of History and Philosophy in Research on Teaching and Learning of Relativity", in International Handbook of Research in History, Philosophy and Science Teaching, edited by M. R. Matthews (Springer, Dordrecht, 2014), pp. 157-181.

[2] C. De Hosson, I. Kermen and E. Parizot, Eur. J. Phys. 31, 1527-1538 (2010).

[3] R. E. Scherr, P. S. Shaffer, and S. Vokos, Am. J. Phys. 69, S24-S35 (2001).

[4] O. Levrini and A.A. diSessa, Physical Review Special Topics - Physics Education Research 4, 010107 (2008).

[5] K. Dimitriadi and K. Halkia, Int. J. Sci. Educ. 34, 25652582 (2012).

[6] P. W. Hewson, Eur. J. Sci. Educ. 4, 61-78 (1982).

[7] J. S. Aslanides and C. Savage, Physical Review Special Topics - Physics Education Research 9, 010118 (2013).

[8] P. C. Peters, Am. J. Phys. 54, 804-808 (1986).

[9] S. Sonego and M. Pin, Eur. J. Phys. 26, 33-45 (2005).

[10] H.Niedderer, M.Budde, D.Givry, D.Psillos, and A. Tiberghien, "Learning process Studies" in Contributions from Science Education Research, edited by R.Pintò and D.Couso (Springer, Dordrecht, 2007), pp. 159-171.

[11] R. T. White and R. F. Gunstone, Probing Understanding (Falmer Press, London, 1992), pp. 1-20.

[12] J. Posner, K. Strike, P. Hewson, and W. Gerzog, Sci. Ed. 66, 211-222 (1982).

[13] W. Bertozzi, Am. J. Phys. 32, 551-555 (1964).

[14] J. K. Gilbert and M. Reiner, Int. J. Sci. Educ. 22, 265283 (2000).

[15] E. Fabri, "Insegnare Relatività nel XXI secolo", AIF 2000 Summer School Proceedings, 2007, pp. 183-190.

[16] E. Taylor and J. Wheeler, Spacetime Physics (Freeman and Company, New York, 1965), pp. 217-219.

[17] R. P. Feynman, Six not-so-easy Pieces (California Institute of Technology Press, 1963), pp. 131-137.

[18] S. Vosniadou, "Conceptual Change Research: an Introduction", in International Handbook of Research on Conceptual Change, edited by S. Vosniadou (Routledge, New York, 2008), pp. xiii-xxviii.

[19] R. Tytler, V. Prain, P. Hubber, and B. Waldrip, Constructing Representations to Learn in Science (Sense Publishers, Rotterdam, 2013), pp. 1-14.

[20] J. K. Gilbert, "Visualization: a Metacognitive Skill in Science and Science Education", in Visualization in Science Education, edited by J. K. Gilbert (Springer, Dordrecht, 2005), pp. 9-27.

[21] P. Mayring, "Qualitative Content Analysis", in $A$ Companion to Qualitative Research, edited by U. Flick, E. von Kardoff and I. Steinke (Sage, London, 2004).

[22] P. Mayring, Forum: Qualitative Social Research 8, Art. 26 (2007).

[23] H. Fischer, W. Boone, and K. Neumann, "Quantitative Research Designs and Approaches" in Handbook of Research on Science Education, edited by N. Lederman and S. K. Abell (Routledge, New York, 2014). 